\title{
ASTHMA IN CYSTIC FIBROSIS
}

\author{
Marcela Daniela Ionescu, Ioana-Alina Anca, Mihaela Balgradean \\ ,Carol Davila" University of Medicine and Pharmacy, Bucharest
}

\begin{abstract}
Cystic fibrosis (CF) is the most common autosomal recessive disease in Caucasians. Lung disease is characterized by impaired mucocilliary clearance with airway obstruction and chronic pulmonary infection and inflammation. Wheeze is a common symptom in CF, but in some cases the wheeze is due to the presence of concomitant asthma. There is no consensus on how to define CF asthma, but the diagnosis is predominantly based on the patient's strong family and personal history of atopy.
\end{abstract}

Keywords: cystic fibrosis, wheeze, asthma, atopy

Cystic fibrosis (CF) is a complex multysistem disease, caused by defects in a single gene. It is the most common autosomal recessive disease in Caucasians associated with early death. Pulmonary manifestations are responsible for most of the morbidity and mortality. (1) CF lung disease begins early in life and is characterized by impaired mucocilliary clearance with airway obstruction, and chronic pulmonary infection and inflammation. Chronic bacterial endobronchial infection is associated with an intense neutrophilic inflammatory response that damages the airway, impairs local host - defence mechanism, and facilitates further infection. This vicious cycle of inflammation and infection with airway damage, result in progressive bronchiectasis, gas trapping, impaired gas exchange and ultimately leads to respiratory failure $(2,3)$.

Wheeze is a common symptom in CF and, whilst in many patients this is due to their underlying lung disease, in some cases the wheeze is due to the presence of concomitant asthma. It is difficult to determine which patients have a combination of asthma and which have asthma like symptoms caused by inflammation of the CF lung. $(3,4)$

The pathogenesis of airway obstruction and wheezing in $\mathrm{CF}$ involves several overlapping mechanisms: airway mucosal oedema secondary to chronic infection and inflammation, mechanical obstruction by abnormal viscid secretions, airway smooth muscle contraction due to stimulation by a host of inflammatory mediators. (5)

Asthma is a common condition but the diagnosis of asthma can be difficult especially in preschool children. International Paediatric Asthma Consensus Group defined asthma as: episodic wheeze and/or cough in a clinical setting where asthma was likely and other rarer conditions have been excluded. This definition is particularly unhelpful in patients with CF. $(6,7)$ Various definitions are used, and common to them all are reversible airway obstruction and chronic airway inflammation. Classical symptoms of asthma are wheeze, cough, dyspnea and chest tightness. For diagnosing asthma are important: history taking, objective documentation of reversible bronchial obstruction, allergy, bronchial hyperesponssiveness (BHR) testing, assessing airway inflammation whenever possible. (7)

It is even harder to define $\mathrm{CF}$ asthma but an attempt has been made by the US and Canadian Epidemiologic Study of Cystic Fibrosis (ESCF). The patient is reported as having concomitant asthma if, in the treating physician's opinion, asthma contributes significantly to the patient's lung disease. The diagnosis of asthma is suggested by the following: episodes of acute airway obstruction reversed by bronchodilators (especially if seasonal); a strong family history of asthma and/or evidence of atopy

Corresponding author:

Marcela Daniela Ionescu, „Carol Davila“ University of Medicine and Pharmacy, 37 Dionisie Lupu St., Bucharest

E-mail: drmarcela.ionescu@gmail.com 
(such as eczema or hay fever); laboratory evidence of allergy such as eosinophillia or elevated IgE. This definition is accepted although the use of serum $\operatorname{IgE}$ and eosinophilia is only of value if allergic bronchopulmonary aspergilosis (ABPA) has been excluded. $(4,6-8)$

\section{HOW MAY CF ASTHMA BE DIAGNOSED?}

Some symptoms are of less relevance to CF patients. For example, a history of wheezing is important but non-specific and most patients with $\mathrm{CF}$ have recurrent coughs whether or not they have asthma. Probably the most appropriate indicators are a family history of atopy (including asthma) and a personal history of eczema and allergic rhinitis. Physical examination is unhelpful, hyperinflation is common in both CF and asthma. $(3,5,6)$ Early physical findings include increased anteroposterior diameter of the chest, generalized hyperresonance, scattered or localized coarse crackles and digital clubbing. Cyanosis is a late sign.

\section{LUNG FUNCTION}

Standard clinic spirometry may indicate the presence of airway obstruction. It does not differentiate obstruction due to concomitant asthma that due to typical CF lung disease. Measurements of small airway function, such as maximal expiratory flow at $25 \%$ vital capacity will not help because they tend to be reduced in most CF patients and are quite variable.

Measuring peak expiratory flow rate are often useful, except in CF where the obstruction mainly involves medium and small airways. Reduction in peakflow is only a late sign. (2-6) Body plethysmography can give further information including the degree of hyperinflation and airway resistance, but it is not specific to CF asthma. $(4,6)$

\section{BRONCHIAL HYPERREACTIVITY (BHR)}

BHR is common in CF patients of all ages. BHR tests are not routinely performed in pediatrics. BHR to histamine or methacholine is found in at least $40 \%$ of patients with CF. $(4,9)$ If bronchial reactivity is measured in CF using challenge testing, many patients exhibit periods of enhanced bronchial reactivity, particularly during and immediately after infective exacerbations. $(1,6,9)$

The airway hyperresponsiveness of CF also differs in its response to histamine induced bronchospasm which is effectively reversed with ipratropi- um in patients with $\mathrm{CF}$ who do not have asthma, whereas ipratropium does not efficiently reverse histamine - induced bronchospasm in those with concomitant symptoms of asthma. These suggest that the increased airway reactivity in patients with $\mathrm{CF}$ is vagally mediated and results from the airway damage caused by the lung disease. (10)

The response to indirect stimuli such as hypertonic saline or exercise is different in $\mathrm{CF}$, because the patients usually bronchodilate after such challenges. (10)

Exercise testing has a important role in evaluating both $\mathrm{CF}$ and asthma. The differences between the response to exercise in the two conditions are important. In asthma, after the normal transient bronchodilatation, progressive bronchoconstriction occurs, and its maximum 5-10 minutes after stopping exercise. Simptoms are usually gone within 15-30 minutes and lung function is back to normal within 30-60 minutes. In CF there is a marked increase in peak expiratory flows during exercise which is most exaggerated in those worst lung disease. After exercise, the decrease in peak flow is seen to a much lesser extent than in asthma. It is probable that the main cause of these results is the instability of the CF airway. $(1,4,6,10,11)$

All patients with CF should be tested to see if their spirometry improves after the use of a bronchodilator (ex. salbutamol, terbutaline or ipratropium bromide) given by aerosol or nebulizer. (11)

Bronchodilator responsiveness is recommended for the diagnosis of asthma, but its usefulness in $\mathrm{CF}$ asthma diagnosis is less obvious. The reason for this is that due to the degree of variability in lung function measures (which can vary by as much as $15-20 \%$ spontaneously, including same day testing). CF patients often show a degree of bronchodilator responsiveness whether or not they have $\mathrm{CF}$ asthma and most studies show no relationship with atopy. $(4,6)$

Hypersensitivity of the airways in CF has long been recognized and many patients with $\mathrm{CF}$ wheeze. This may be due to bronchospasm, mucosal edema or retained secretions in the airways. Once allergic bronchopulmonary aspergillosis and gastroesofageal reflux have been excluded we can use medication for this. $(4,6,11)$

Patient with CF have concomitant asthma are manifested by recurrent acute symptoms of dyspnea that is impressively responsive to an inhaled 32-agonist and/or systemic corticosteroids (8). However, many others have a degree of bronchodilator responsiveness in the absence of an good clinical response to such antiasthmatic treatment. In 
contrast to those with asthma, exercise does not induce bronchospasm and can even cause bronchodilatation in patients with CF who have bronchodilator responsiveness in the absence of asthma. $(4,6$, 8-10)

\section{ATOPY}

Serum total IgE can be a guide to patient's atopic status. Positive skin prick testing or serum radioallergosorbent tests (RAST) will indicate atopic status when the common aeroallergens such as house dust mite, cat, dog, grass and tree pollens, are tested. A reaction to Aspergillus does not necesarly denote atopy. It should not therefore form part of atopic screening in $\mathrm{CF}$. The prevalence of atopy in $\mathrm{CF}$ is in fact similar to that in the non CF population. (1-6) It has been stated that atopic CF patients have worse lung disease.

\section{DIFFERENTIAL DIAGNOSIS}

In a $\mathrm{CF}$ patient with intractable wheezing certain condition need to be excluded and form part of the differential diagnosis of CF asthma. They can coexist too.

Tracheo-bronchomalacia needs to be excluded by flexible bronchoscopy. The importance of this diagnosis is that it can mimic asthma symptoms, but may be worsened by bronchodilators relaxing the smoth muscle.

Gastro-oesophageal reflux is common in infants with $\mathrm{CF}$, but the problem persist in many older children and adults. It is well established that reflux is associated with bronchospasm and wheezing. A 24 hour ph study needs to be performed to confirm the diagnosis. $(3,5)$

Allergic bronchopulmonary aspergillosis must be excluded, although sometimes the diagnosis is difficult. The classical case is easy to diagnose. However many of the symptoms and signs of ABPA are common and mimic CF lung disease. Cystic Fibrosis Foundation consensus Conference proposed criteria for classic ABPA, minimal diagnostic criteria and recommendations for screening $(12,13)$.

Severe small airways disease is difficult to define precisely. Children have intractable wheezing and often sputum expectoration is minimal even though the lungs are full of secretions. High resolutions computer tomography (HRCT) may be useful in patient who are not responding to standard treatments. It reveals extensive small airways disease manifest by distal air trapping due to fixed obliterative bronchiolitis. $(4,6,11,12)$

\section{TREATMENT}

Many patients with CF exacerbations also have airway hyperresponsiveness and an improvenment in lung function in hospitalized patients has been shown with regular nebulized salbutamol. $\beta$-adrenergic agoniste enhance ciliary beat frequency and may affect mucus secretion. $(11,14,15)$ Terbutaline has been shown to stimulate chloride ion secretion in the lumen and may increase hydration of the airway. (10) Theophylline increases mucociliary clearance, diaphragmatic contractility and central nervous system drive to the respiratory centers, and may also be anti-inflammatory, at least in the context of asthma in the non CF patient. Some studies have shown theophylline to be beneficial in CF asthma. (10)

Inhaled corticosteroid are very widely prescribed in CF, often without much justification. Infants are sometime prescribed them prior to diagnosis because their symptoms have been attributed to asthma. Another reason inhaled steroids are prescribed to known CF patients is the mistaken belief that the noise the patient or family is describing is related to bronchospasm, not airway secretions. Moust would believe that giving inhaled steroids to reduce $\mathrm{CF}$ - related inflammation is not a correct strategy. Therefore, the only indication for using them in CF is if the patient has asthma. $(1,11,14)$

A stepwise treatment approach for asthma has been proposed by all international guidelines. In patients with uncontrolled asthma, step-up treatment should be considered. (7) In patients with CF asthma the treatment follows the standard stepwise progression used in asthma: short-acting bronchodilator, inhaled corticosteroid, long-acting $\beta$ agonist, theophyllines (which may aid mucociliary clearance), leukotriene receptor antagonist (limited evidence in $\mathrm{CF}$, but may decrease eosinophilic inflammation), oral steroids. The response to antiasthma therapy in an individual may be as helpful in the diagnosis of CF asthma $(4,6,7,10,14-16)$

\section{CONCLUSION}

There is no consensus on how to define CF asthma. The overlap between the symptoms of CF lung disease and those of asthma, and the fact that both condition are characterized by bronchial reactivity, variable airway obstruction and infective exacerbations, complicates the precise diagnosis of asthma in $\mathrm{CF}$.

Factors that support diagnosis of cystic fibrosis asthma are: history of recurrent wheezing, positive 
family history atopy, patient s history of atopy (eczema, hay fever,food allergy), positive skin prick tests or serum radioallergosorbent tests (RAST) to aeroallergens (excluding Aspergillus), high serum IgE (excluding ABPA). Pulmonary function tests (spirometry, bronchodilator responsiveness, BHR), other diagnose excluded (gastroesophageal reflux, malacic airways, ABPA, obliterative bronchiolitis) and the response to antiasthma medication may also help the diagnosis. $(4,6,7)$

However it still be difficult to determine who has $\mathrm{CF}$ and asthma and who has asthma like symptoms of prolonged exhalation, wheeze, and crackles due to underlying lung inflammation. A analysis

\section{REFERENCES}

1. Horsley A. Genetics and pathophysiology. Cystic fibrosis, Oxford Resp. Med., 2010; 1-17

2. Cunningham S. Diagnosis and process of care. Cystic fibrosis, Oxford Resp. Med., 2010; 18-31

3. Regamey N., Barben J. CF lung disease. Paediatric Resp Med., European Resp Soc., 2013; 402-410

4. Balfour-Lynn I.M., Elborn J.S. CF-asthma: what is it and what do we do about it? Thorax 2002 Aug; 57(8);742-8

5. Schechter M.S., O'Sullivan B.P. Cystic fibrosis, Pediatric Pulmonology, American Academy of Pediatrics, 2011; 717-745

6. Balfour Lynn I.M. Asthma in cystic fibrosis. J.R.Soc.Med 2003, 96 Suppl 43; 30-4

7. Pijnenburg M., Carlsen C.L. Bronchial asthma. Paediatric Resp. Med., European Resp Soc., 2013; 316-334

8. Keeley D., Mc Keen M. Asthma and other wheezing disorders in children. Clin Evid 2005 Dec; (14); 238-62

9. Chapman S., Robinson G., Stradling J. Cystic fibrosis, Oxford Handbook of Respiratory Medicine 2012; 205-227 from the North American and European databases, it seems that labeling a CF patients with concomitant asthma influenced drug prescribing. $(4,6)$

Identifying patients with significant co-existing asthma is, however, important as they may benefit from specific asthma medications, in particular inhaled steroids. (11)

Whether wheezing is due to concomitant asthma or not, the treatment follows the standard step-wise progression used in asthma.

Whatever the therapy prescribed by the physician, it should only be continued if the benefits are objectively proven.
10. Parker A.L. Airway reactivity is a determinant of bronchodilator responsiveness after methacholine-induced bronchoconstriction. J Asthma. 2004 Sep; 41(6):671-7.

11. Hodson M., Bush A. Respiratory disease, Cystic fibrosis, Third edition 2007; 159 - 175

12. Bush A. Allergic bronchopulmonary aspergillosis. Paediatric Resp. Med., European Resp Soc., 2013; 376 - 383

13. Kravitz R.M. Allergic bronchopulmonary aspergillosis. Pediatric Pulmonology, American Academy of Pediatrics, 2011; 187-209

14. Brand P.L.P. Bronchodilators in cystic fibrosis. J.R. Soc. Med. 2000; 93(suppl 38):37-9

15. Colombo J.L. Long-acting bronchodilators in cystic fibrosis. Curr Opin Pulm Med. 2003 Nov; 9(6):504-8.

16. Schmitt-Grohe S., Zielens S. Leukotriene receptor antagonists in children with cystic fibrosis lung disease: antinflammatory and clinical effects. Paediatric Drugs 2005; 7(6):353-63 